\title{
Coupled-resonator optical waveguides: Q-factor influence on slow-light propagation and the maximal group delay
}

Søren Raza

Jure Grgić

Jesper Goor Pedersen

\section{Sanshui Xiao}

Niels Asger Mortensen asger@mailaps.org

\begin{abstract}
Department of Photonics Engineering, DTU Fotonik, Technical University of Denmark, DK-2800 Kongens Lyngby, Denmark

Department of Photonics Engineering, DTU Fotonik, Technical University of Denmark, DK-2800 Kongens Lyngby, Denmark

Department of Photonics Engineering, DTU Fotonik, Technical University of Denmark, DK-2800 Kongens Lyngby, Denmark

Department of Photonics Engineering, DTU Fotonik, Technical University of Denmark, DK-2800 Kongens Lyngby, Denmark

Department of Photonics Engineering, DTU Fotonik, Technical University of Denmark, DK-2800 Kongens Lyngby, Denmark
\end{abstract}

Coupled-resonator optical waveguides hold potential for slow-light propagation of optical pulses. The dispersion properties may adequately be analyzed within the framework of coupled-mode theory. We extend the standard coupled-mode theory for such structures to also include complex-valued parameters which allows us to analyze the dispersion properties also in presence of finite $Q$ factors for the coupled resonator states. Near the band-edge the group velocity saturates at a finite value $v_{g} / c \propto \sqrt{1 / Q}$ while in the band center, the group velocity is unaffected by a finite $Q$ factor as compared to ideal resonators without any damping. However, the maximal group delay that can be envisioned is a balance between having a low group velocity while not jeopardizing the propagation length. We find that the maximal group delay remains roughly constant over the entire bandwidth, being given by the photon life time $\tau_{p}=Q / \Omega$ of the individual resonators. [DOI: 10.2971/jeos.2010.10009]

Keywords: coupled-resonator optical waveguide (CROW), photonic crystal waveguides, slow light, group delay

\section{INTRODUCTION}

The coupled-resonator optical waveguide (CROW) was first proposed and analyzed by Yariv et al. [1]. One particularly interesting property is that CROWs in principle offer slowlight propagation. In a simple picture, the group velocity is low when the wave package will dwell for a long time in one resonator before tunneling onto the next resonator and so on. Of course, to take full advantage of the CROW concept, the quality factor $Q$ should be sufficiently high that the photon life time $\tau_{p}$ of an isolated resonator much exceeds the tunneling time $\tau_{t}$ in which case the group velocity will be of the order $v_{g} \sim a / \tau_{t}$ with $a$ being the spacing of the resonators. The original work emphasized the coupling of ideal and identical resonators [1] and more recently the influence of disorder on the group velocity has been studied in detail [2]-[4]. CROWs have been proposed and realized in a number of ways, utilizing for example, high-Q microspheres [5], ring resonators [6, 7], or defect cavities in photonic crystals [8]-[13]. There is a general consensus that resonators with an intrinsic high $Q$ are needed, but according to our knowledge the influence of a finite $Q$ has not yet been analyzed in detail with respect to the interplay of slow-light and damping. In this work we explicitly account for a finite intrinsic quality factor of the resonators forming the CROW. Broadening of van Hove singularities in photonic crystal waveguides limits the slow down near band edges [14] and for the CROWs we find a similar effect which can be studied explicitly within the framework of coupled mode theory. Most importantly we find that when treating slow light and damping on an equal footing, damping is jeopardizing some of the attractive features of the slow-light propagation. In the following we first review the derivation of a general expression for the dispersion relation (see Section 2) and subsequently we derive analytical expressions for $Q$-factor dependence of the group velocity near the band edges as well as in the center of the band (see Section 3). Furthermore, we discuss the maximal group delay that one may achieve with CROWs (see Section 4) and as an example we apply the coupled-mode formalism to a photonic crystal waveguide structure (see Section 5). Finally, conclusions are given (see Section 6).

\section{COUPLED-MODE THEORY}

Consider a resonator with a resonant field

$$
\mathbf{E}_{j}(\mathbf{r}, t)=\mathbf{E}_{j}(\mathbf{r}) \exp \left[i\left(\Omega_{j}+i \delta \Omega_{j} / 2\right) t\right]
$$

so that the energy in the resonator $\left|\mathbf{E}_{j}(\omega)\right|^{2}$ has a Lorentzian frequency distribution corresponding to the density-of-states

$$
\rho_{j}(\omega)=\frac{1}{\pi} \frac{\delta \Omega_{j} / 2}{\left(\omega-\Omega_{j}\right)^{2}+\left(\delta \Omega_{j} / 2\right)^{2}}
$$


where $\Omega_{j}$ is the resonance frequency, $\delta \Omega_{j}$ is the resonance line width, and $Q_{j}=\Omega_{j} / \delta \Omega_{j}$ is the quality factor of the $j$ th resonator, corresponding to a photon life time $\tau_{p}=Q / \Omega$.

Next, imagine a chain of coupled resonators of the above kind. We follow the work of Yariv and co-workers [1] and write the electrical field as a linear combination of the isolated resonator fields $\mathbf{E}_{j}$, while allowing for complex-valued parameters, like the resonance frequency $\Omega_{j}+i \delta \Omega_{j} / 2$ and the coupling elements $\gamma_{j+1, j}$. We further consider the case where the resonators are all identical and arranged in a fully periodic sequence with nearest-neighbor coupling only. For clarity we may thus suppress all indices.

The electromagnetic states now form a continuous band with a dispersion relation (see e.g. $[3,15]$ )

$$
\omega(\kappa)=\Omega\left(1+i \frac{1}{2 Q}\right)\left(1-\frac{\Delta \gamma}{2}-\gamma \cos (\kappa a)\right)
$$

where $\kappa=\kappa^{\prime}+i \kappa^{\prime \prime}$ is the complex valued Bloch wave vector and $a$ is the lattice constant of the periodic arrangement of resonators. On the right-hand side,

$$
\gamma=\left\langle\mathbf{E}_{j}|\Delta \epsilon| \mathbf{E}_{j+1}\right\rangle
$$

is the coupling term of two neighboring resonators $j$ and $j+1$, while

$$
\Delta \gamma=\left\langle\mathbf{E}_{j}|\Delta \epsilon| \mathbf{E}_{j}\right\rangle
$$

is the small lowering of the isolated resonance frequency caused by the presence of neighboring resonators. In the framework of the tight-binding model, this is referred to as the lowering of the 'on-site' energy. Here, we have assumed that the fields are normalized so that $\left\langle\mathbf{E}_{n}\left|\epsilon_{n}\right| \mathbf{E}_{n}\right\rangle=\int \epsilon_{n}(\mathbf{r}) \mathbf{E}_{n}^{*}(\mathbf{r})$. $\mathbf{E}_{n}(\mathbf{r})=1$, where $\epsilon_{n}(\mathbf{r})$ is the dielectric function of the isolated resonator. Eq. (3) is a generalization of the theory by Yariv et al. [1] to also include resonators with a finite $Q$-factor. Potentially, $\gamma$ and $\Delta \gamma$ may also be complex, for example in the presence of material absorption, but for simplicity we will treat $\gamma$ as a real parameter here.

\section{DISPERSION, GROUP VELOCITY, AND DENSITY-OF-STATES}

The group velocity may formally be calculated from the dispersion relation in Eq. (3). Keeping in mind that $\omega$ is real while $\kappa$ may be complex, we have that the group velocity is given by

$$
v_{g}=\frac{1}{\operatorname{Re}\{\partial \kappa / \partial \omega\}}
$$

Isolating $\kappa$ in Eq. (3) we get $\kappa=(1 / a) \arccos [f(\omega)]$ which may formally be differentiated to give $\partial \kappa / \partial \omega=-(1 / a)[1-$ $\left.f^{2}(\omega)\right]^{-1 / 2} \partial f / \partial \omega$, with

$$
f(\omega) \equiv \frac{1}{\gamma}\left(1-\frac{\Delta \gamma}{2}-\frac{2 Q \omega}{\Omega(2 Q+i)}\right) .
$$

Taking the inverse of the real part we then arrive at an analytical expression for the group velocity. Though the calculation is straightforward, the final result is lengthy and it is not reproduced here. Along the same lines, we may also calculate

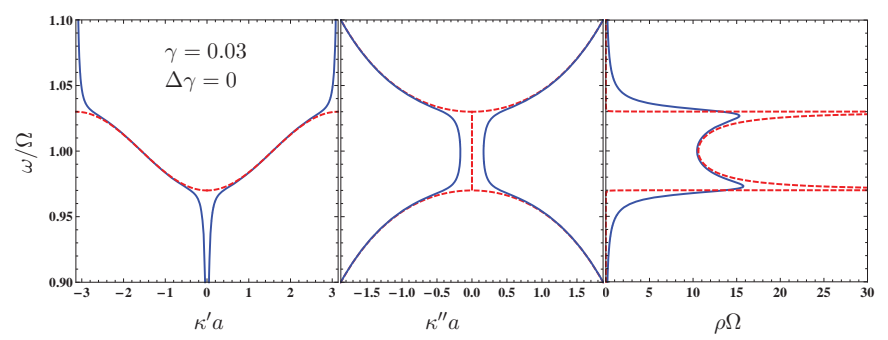

FIG. 1 Complex dispersion relation for a CROW. Dashed lines are for $Q=\infty$ while solid lines correspond to $Q=10^{2}$. The left panel shows the frequency $\omega$ versus the real part of the Bloch wave vector $\kappa^{\prime}$, the middle panel shows the frequency $\omega$ versus the imaginary part of the Bloch wave vector $\kappa^{\prime \prime}$, and the right panel shows the density-of-states $\rho(\omega)$.

density-of-states from the dispersion relation in Eq. (3). For the particular case of a one-dimensional chain, the density-ofstates is inversely proportional to the group velocity, giving rise to the following density-of-states

$$
\rho(\omega)=\frac{a}{\pi} \operatorname{Re}\left\{\frac{\partial \kappa}{\partial \omega}\right\} .
$$

Figure 1 illustrates the dispersion properties of the CROW. The left panel illustrates the relation between the frequency $\omega$ and the real part $\kappa^{\prime}$ of the complex-valued Bloch wave vector $\kappa=\kappa^{\prime}+i \kappa^{\prime \prime}$. Likewise, the middle panel illustrates the relation between frequency $\omega$ and the imaginary part $\kappa^{\prime \prime}$ of the Bloch wave vector. Finally, the right panel shows the corresponding density-of-states. The difference between the ideal structure $(Q \rightarrow \infty)$ and one employing resonators of finite $Q$ is contrasted by the dashed and solid lines, respectively. Notice how the finite $Q$ factor serves to smear out van Hove singularities in the density-of-states. In the dispersion relation this has its counterpart in the group velocity not going to zero near the high-symmetry points corresponding to the band edges of the ideal structure. Also, quite steep bands appear outside the traditional band of extended states, though of course with a significant attenuation as evident from the middle plot illustrating the $\kappa^{\prime \prime}$ dependence.

In the following we analyze the result at the band-center and the band-edges in more detail. For simplicity we assume $\gamma \ll$ 1 , which is also the relevant regime for slow-light applications. Furthermore, we neglect the small shift $\Delta \gamma$ so that the band is centered around $\omega=\Omega$ with band-edges at $\omega=(1 \pm \gamma) \Omega$. At the band center we get

$$
\frac{v_{g}}{v_{0}}=1+\frac{1}{8} \frac{1}{\gamma^{2} Q^{2}}+\mathcal{O}\left(Q^{-4}\right), \quad \text { (band center) }
$$

where $v_{0}=\gamma a \Omega$ is the group velocity at the band center for infinite- $Q$ resonators. Likewise, at the band-edges we get

$$
\frac{v_{g}}{v_{0}}=\sqrt{\frac{2}{|\gamma| Q}}+\mathcal{O}\left(Q^{-3 / 2}\right) . \quad \text { (band edges) }
$$

The first result illustrates that in the center of the band, the group velocity is rather insensitive to the quality factor, and given by $v_{0}$ provided that $Q \gg 1 / \gamma$. On the other hand, at the band edges the group velocity scales quite unfavorably with the $Q$ factor, making the slow-light regime challenging 


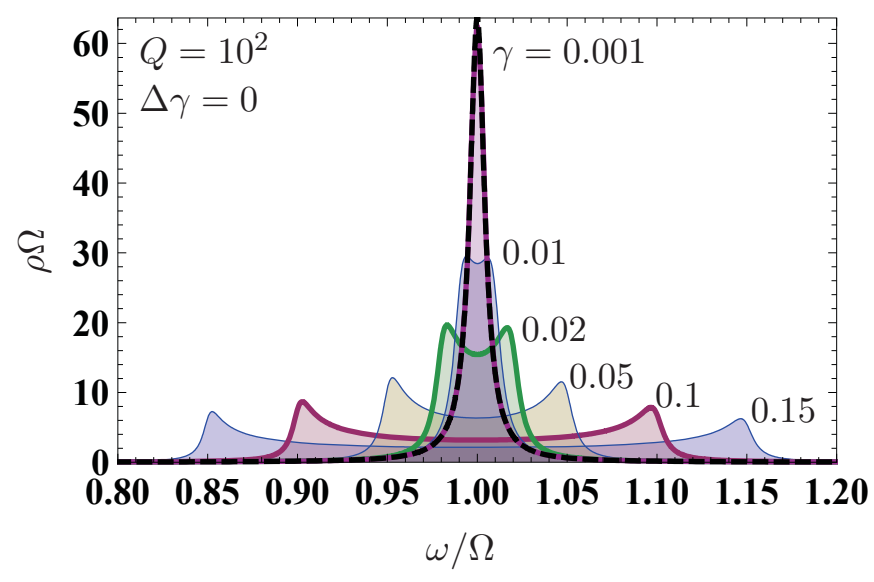

FIC. 2 Density-of-states for a CROWs with varying coupling, Eq. (8). The dashed line illustrates the Lorentzian density-of-states for the uncoupled resonator, Eq. (2).

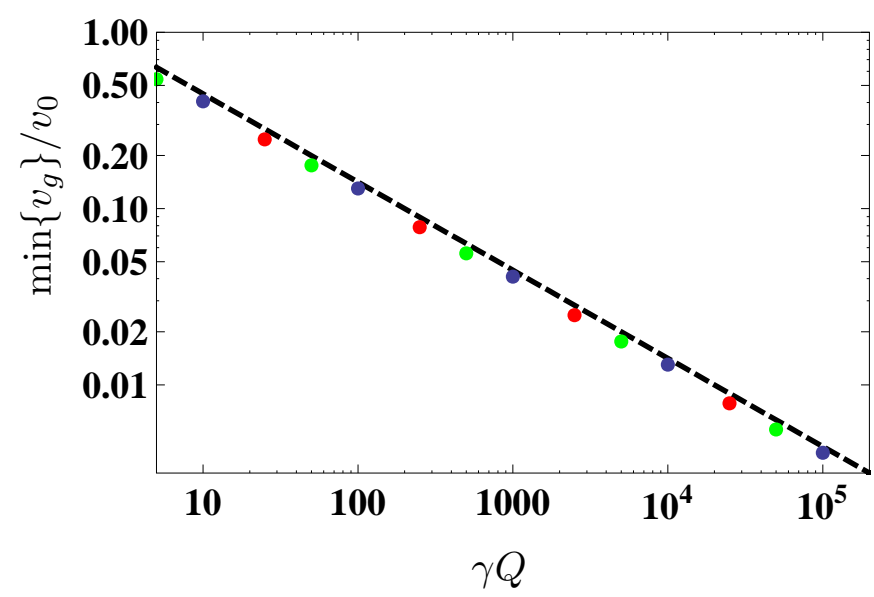

FIG. 3 Plot of the minimum group velocity versus $\gamma Q$ calculated numerically from Eq. (6) for the three different cases of $\gamma=0.001,0.01$, and 0.1. The dashed line shows the asymptotic expression for the group velocity at the band edge, Eq. (10).

to explore. In the case of absorption, the quality factor $Q_{\mathrm{abs}}$ is inversely proportional to the imaginary part $\epsilon^{\prime \prime}$ of the dielectric function $[16,17]$ so that $v_{g} \propto \sqrt{\epsilon^{\prime \prime}}$ in full agreement with the perturbative results derived from band-structure considerations in [14]. At the band center, the group velocity of the ideal CROW is finite and given by $v_{0}$. Furthermore, the group velocity is hardly dispersive (the second-order derivative is small), thus making the group velocity itself quite insensitive to dissipation [18]. Results similar to Eqs. (9) and (10) were reported recently in [3].

The interplay of the coupling strength $\gamma$ and the quality factor $Q$ is also illustrated in Figure 2, depicting how the initial Lorentzian line-shape broadens with increasing $\gamma$ into a band with van Hove singularities at the two band edges. Notice how the area below the curves is conserved. Obviously, the most pronounced slow down occurs at the van Hove singularities associated with band edges. The smearing by the finite $Q$ serves to shift the minimum in the group velocity slightly away from the band edge. Figure 3 shows the minimum group velocity versus $\gamma Q$ calculated numerically from Eq. (6) for the three different cases of $\gamma=0.001,0.01$, and 0.1 . As seen, the full results are in excellent agreement with the predictions of Eq. (10) shown by the dashed line.

\section{THE MAXIMAL GROUP DELAY}

The group delay is given by $\tau=L / v_{g}$ with $L$ being the length of the waveguide. To estimate the maximal realistic group delay we use that for any practical purpose $L \lesssim 1 / \alpha$ with $\alpha=2 \kappa^{\prime \prime}$ being the damping parameter. This gives an upper bound

$$
\tau_{\max } \sim \frac{1}{v_{g} \alpha}=\frac{1}{2 \kappa^{\prime \prime}} \frac{\partial \kappa^{\prime}}{\partial \omega} .
$$

Combining the full results for $v_{g}$ and $\alpha$ and expanding in $1 / Q$ we get

$$
\tau_{\max } \sim \frac{Q}{\Omega}+\mathcal{O}\left(Q^{-1}\right)=\tau_{p}+\mathcal{O}\left(Q^{-1}\right) .
$$

Quite intuitively, the maximal group delay is limited by the photon life time $\tau_{p}=Q / \Omega$ of the individual resonators independently on the actual frequency. Despite the reduced group velocity near the band edges, the advantage of a slowly advancing wave package is balanced by a reduced propagation length, see the middle panel of Figure 1. According to our knowledge, this is an overlooked issue which is important for the potential application of CROW concepts in optical buffers and delay-line architectures. We emphasize that compared to a single resonator, the $\mathrm{CROW}$ of course offers the advantage of an increased bandwidth.

\section{PHOTONIC CRYSTAL EXAMPLE}

Finally, we consider a CROW realized by coupled defects in a photonic crystal. For simplicity, we consider a twodimensional photonic crystal with triangular lattice of airholes of diameter $d$ and pitch $\Lambda$. By removing every third air hole on a line we form a CROW with a lattice constant $a=3 \Lambda$. In order to compare the predictions of Eq. (3) to full-vector simulations we employ a plane-wave method [19]. We consider air holes of diameter $d=0.6 \Lambda$ in a dielectric material with $\epsilon=7.0225$, and using a super-cell approximation the plane-wave method gives $\Omega \Lambda / 2 \pi c=0.3079$ for the resonance frequency of an isolated defect cavity. For the corresponding CROW we obtain the dispersion relation indicated by data points in Figure 4 . The dashed line shows a least-square error fit to Eq. (3) with $\Omega \Lambda / 2 \pi c=0.3074$, $\Delta \gamma=3.705 \times 10^{-5}$, and $\gamma=-0.0066$, while $Q \rightarrow \infty$. Note how the fitted value of $\Omega$ agrees excellently with the value obtained independently for the isolated defect cavity. Furthermore, the parameters indeed satisfy $\Delta \gamma \ll \gamma \ll 1$ as assumed in our analysis leading to Eqs. (9) and (10). Consequences of a finite $Q$ factor can now immediately be predicted and the solid line shows how the dispersion changes in the presence of a finite quality factor, $Q=10^{3}$. At the band edges, Eq. (10) in this particular case leads to a maximal group index of $n_{g} \sim(3 / 2) \sqrt{Q}$, so that $Q=10^{4}$ would allow a group index up to $n_{g} \sim 150$, while $Q=10^{3}$ would limit the group index to $n_{g} \sim 50$. However, as discussed above the high group indices do not come for free as they will be associated with an increased damping.

\section{CONCLUSION}

In conclusion, we have derived an explicit relation for the dispersion relation of $\mathrm{CROW}$ made from resonators with a finite 


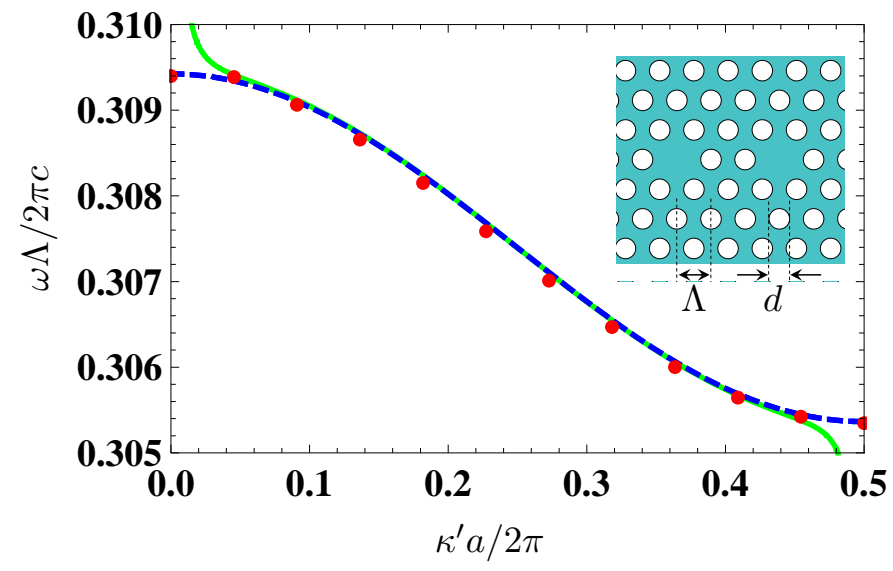

FIG. 4 Dispersion relation for a CROW in a two-dimensional photonic crystal with $\epsilon=$ 7.0225 and with air-hole diameter $d=0.6 \Lambda$ and waveguide pitch $a=3 \Lambda$, with $\Lambda$ being the pitch of the air-hole lattice. Data points are obtained with a plane-wave method [19] while the dashed line shows Eq. (3) with $\Omega \Lambda / 2 \pi c=0.3074, \Delta \gamma=$ $3.705 \times 10^{-5}$, and $\gamma=-0.0066$, and $Q \rightarrow \infty$. The solid line shows corresponding results for the case of $Q=10^{3}$.

$Q$ factor. A finite $Q$ profoundly influences the van Hove singularities near the band edges with a resulting limitation of the group index while at the center of the band the dispersion properties are less affected. Simple analytical expressions are supported by calculations of the group velocity, demonstrating how the $Q$ enters on an equal footing with the coupling $\gamma$ corresponding to the competing time scales associated with photon decay and tunneling. In the context of practical applications involving the group delay, we note that the maximal attainable group delay appears as a balance between the reduced group velocity and the the decay length. Explicit calculations show that irrespectively of the underlying bandstructure, the maximal group delay is limited by the photon life time of the resonators. This illustrates the importance of addressing propagation loss and slow-light on an equal footing.

\section{ACKNOWLEDGEMENTS}

This work is financially supported by the Villum Kann Rasmussen Centre of Excellence NATEC (Nanophotonics for Terabit Communications).

\section{References}

[1] A. Yariv, Y. Xu, R. K. Lee, and A. Scherer, "Coupled-resonator optical waveguide: a proposal and analysis" Opt. Lett. 24, 711-713 (1999).

[2] S. Mookherjea, and A. Oh, "Effect of disorder on slow light velocity in optical slow-wave structures" Opt. Lett. 32, 289-291 (2007).

[3] D. P. Fussell, S. Hughes, and M. M. Dignam, "Influence of fabrica- tion disorder on the optical properties of coupled-cavity photonic crystal waveguides" Phys. Rev. B 78, 144201 (2008).

[4] C. Ferrari, F. Morichetti, and A. Melloni, "Disorder in coupledresonator optical waveguides" J. Opt. Soc. Am. B 26, 858-866 (2009).

[5] D. H. Broaddus, M. A. Foster, I. H. Agha, J. T. Robinson, M. Lipson, and A. L. Gaeta, "Silicon-waveguide-coupled high-Q chalcogenide microspheres" Opt. Express 17, 5998-6003 (2009).

[6] F. N. Xia, L. Sekaric, M. O’Boyle, and Y. Vlasov, “Coupled resonator optical waveguides based on silicon-on-insulator photonic wires" Appl. Phys. Lett. 89, 041122 (2006).

[7] A. Melloni, F. Morichetti, C. Ferrari, and M. Martinelli, "Continuously tunable 1 byte delay in coupled-resonator optical waveguides" Opt. Lett. 33, 2389-2391 (2008).

[8] S. Olivier, C. Smith, M. Rattier, H. Benisty, C. Weisbuch, T. Krauss, R. Houdré, and U. Oesterle, "Miniband transmission in a photonic crystal coupled-resonator optical waveguide" Opt. Lett. 26, 10191021 (2001).

[9] T. J. Karle, D. H. Brown, R. Wilson, M. Steer, and T. F. Krauss, "Planar photonic crystal coupled cavity waveguides" IEEE J. Sel. Top. Quant. 8, 909-918 (2002).

[10] H. Altug, and J. Vuckovic, "Two-dimensional coupled photonic crystal resonator arrays" Appl. Phys. Lett. 84, 161-163 (2004).

[11] H. Altug, and J. Vuckovic, "Experimental demonstration of the slow group velocity of light in two-dimensional coupled photonic crystal microcavity arrays" Appl. Phys. Lett. 86, 111102 (2005).

[12] A. Martinez, J. Garcia, P. Sanchis, E. Cuesta-Soto, J. Blasco, and J. Marti, "Intrinsic losses of coupled-cavity waveguides in planarphotonic crystals" Opt. Lett. 32, 635-637 (2007).

[13] S. Vignolini, F. Intonti, M. Zani, F. Riboli, D. S. Wiersma, L. H. Li, L. Balet, M. Francardi, A. Gerardino, A. Fiore, and M. Gurioli, "Near-field imaging of coupled photonic-crystal microcavities" Appl. Phys. Lett. 94, 151103 (2009).

[14] J. G. Pedersen, S. Xiao, and N. A. Mortensen, "Limits of slow light in photonic crystals" Phys. Rev. B 78, 153101 (2008).

[15] D. P. Fussell, and M. M. Dignam, “Engineering the quality factors of coupled-cavity modes in photonic crystal slabs" Appl. Phys. Lett. 90, 183121 (2007).

[16] N. A. Mortensen, S. S. Xiao, and J. Pedersen, “Liquid-infiltrated photonic crystals: enhanced light-matter interactions for lab-on-achip applications" Microfluid. Nanofluid. 4, 117-127 (2008).

[17] T. Xu, M. S. Wheeler, H. E. Ruda, M. Mojahedi, and J. S. Aitchison, "The influence of material absorption on the quality factor of photonic crystal cavities" Opt. Express 17, 8343-8348 (2009).

[18] J. Grgić, J. G. Pedersen, S. Xiao, and N. A. Mortensen, "Groupindex limitations in slow-light photonic crystals" article in press at Photonics Nanostruct. (2009)

[19] S. G. Johnson, and J. D. Joannopoulos, "Block-iterative frequencydomain methods for Maxwell's equations in a planewave basis" Opt. Express 8, 173-190 (2001). 